\title{
Actitudes de alumnos de primaria ante la promoción automática desde la experiencia docente
}

\author{
Luz Marisa Alcocer Escalante \\ marisaalcocer96@gmail.com \\ Universidad Autónoma de Yucatán \\ Mérida, México \\ Ángel Martín Aguilar Riveroll \\ aaguilar@correo.uady.mx \\ Universidad Autónoma de Yucatán \\ Mérida, México
}

\section{RESUMEN}

La normativa de Promoción Automática genera diferentes actitudes en los estudiantes, siendo favorecedoras como la responsabilidad y el compromiso, o negativas como la apatía y el desinterés; casi siempre, la mayor parte de estas actitudes son generadas en los alumnos por los mismos padres o tutores. Es así, que el propósito del trabajo es dar a conocer las actitudes que desarrollan los estudiantes promovidos a causa de la modalidad de Promoción Automática desde la experiencia docente de una escuela primaria de Mérida, México. Se realizó un estudio cualitativo con la técnica de estudio de caso singular descriptivo en la que a través de entrevistas semi estructuradas, nueve actores educativos, expusieron sus percepciones acerca del desenvolvimiento del alumnado que se encuentra protegido por tal normativa. Actitudes como el desinterés y apatía hacia el aprendizaje, así como el miedo al recursamiento, fueron las más señaladas. Se sugiere para futuras investigaciones, recopilar la información directamente con los estudiantes a través de las mismas técnicas o mediante la observación.

Palabras clave: promoción automática; actitud; educación formal; acreditación; percepción. 


\title{
Attitudes of primary school students to automatic promotion from the teaching experience
}

\begin{abstract}
The Automatic Promotion norm generates different attitudes in the students, being favorable such as responsibility and commitment, or negative such as apathy and disinterest; almost always, most of these attitudes are generated in the students by their parents or tutors. Thus, the purpose of this work is to make known the attitudes developed by students promoted because of the Automatic Promotion modality from the teaching experience of an elementary school in Merida, Mexico. A qualitative study was conducted with the technique of descriptive singular case study in which through semi-structured interviews, nine educational actors, exposed their perceptions about the development of students who are protected by such regulations. Attitudes such as disinterest and apathy towards learning, as well as fear of recourse, were the most frequently mentioned. For future research, it is suggested to collect information directly from students through the same techniques or through observation.
\end{abstract}

Keywords: automatic promotion; attitude; formal education; accreditation; perception

Artículo recibido: 30 noviembre. 2021 Aceptado para publicación: 29 diciembre 2021 Correspondencia: marisaalcocer96@gmail.com Conflictos de Interés: Ninguna que declarar 


\section{INTRODUCCIÓN}

Se dice que las actitudes de una persona determinan la aceptación o apatía ante determinadas situaciones. Para el caso de los niños el contexto que viven en el hogar y la manera en la que los padres o tutores hacen frente a las dificultades, será la forma en la que ellos probablemente a futuro decidan enfrentarlos o ignorarlos (Ortega et al., 2010). De la misma manera, funciona con las posturas $\mathrm{u}$ opiniones de temas $\mathrm{y}$ acciones específicas, las cuales se ven reflejadas en los comportamientos o comentarios de los infantes, pues recuperan todo lo que escuchan o ven en casa y principalmente fijándose de lo que hacen sus padres de familia. Tal es la situación que se vive en México con los padres de familia ante la normativa de la Promoción Automática (PA) la cual direcciona la acreditación de los alumnos con el simple hecho de estar inscritos en un grado escolar, por lo tanto, no contempla los saberes y habilidades adquiridos. En consecuencia, aquello que los padres comentan o refieren de la norma, lo transmiten a los hijos y ellos lo reflejan en la escuela con sus maestros y compañeros.

Parte de las posturas que la modalidad de acreditación ha desarrollado en los padres de familia engloban apatía, desinterés e irresponsabilidad hacia los estudios de sus hijos, pues saben que con el hecho de estar inscritos y sin asistir a la escuela, los alumnos pueden pasar de un primer grado a un segundo, por ejemplo. Razeto (2016) menciona que aquellos niños cuyos padres se involucran más con la escuela, son aquellos que tienen mejores actitudes, que aquellos que no lo hacen. Por la otra parte, los padres de familia que no participan de las actividades escolares o no muestran interés por la formación de sus hijos, son aquellos cuyos hijos manifestarán comentarios o actitudes poco favorables hacia sus estudios (Delgado, 2019) lo cual transmitirá a los educandos las mismas actitudes de rechazo y falta de compromiso escolar.

Académicamente los resultados que se tienen sobre efectos que genera la PA en los discentes son escasos (Koppensteiner, 2011) y tratándose de las actitudes, la evidencia empírica es aún más ausente. Los efectos que esta normativa causa son poco claros (Vallejo-García, 2018), lo que no permite contar con un bagaje conciso de las actitudes que los estudiantes de primaria pueden manifestar ante la PA. A partir de las creencias docentes, se puede llegar a experimentar desde la desmotivación en el alumnado (Jimerson, et al. 2006), hasta las actitudes violentas en aquellos estudiantes promovidos (Rathman, et al. 2020). No obstante, aún hace falta trabajo que dé a conocer estos aspectos 
que en ocasiones el sistema educativo no contempla que los estudiantes manifiesten, pues el hacer un esfuerzo porque el alumno no abandone sus estudios como lo sugiere la PA, puede continuar incrementando en las nuevas generaciones la cultura del conformismo y apatía que por años ha caracterizado a los mexicanos tal como lo plasmó Octavio Paz en su "Laberinto de la soledad".

Es por ello que el propósito de este trabajo es dar a conocer las actitudes que desarrollan los estudiantes que promueven a causa de la política de PA desde la opinión docente, pues son estos actores educativos que tienen la oportunidad de mirar de cerca el desempeño y comportamiento de los alumnos en el aula.

\section{DESARROLLO}

\section{La política de Promoción Automática}

Esta normativa de acreditación surge con la intención de aminorar el abandono escolar por parte de los alumnos, pues en países como México, Uganda y Camerún, se estaba convirtiendo en una situación desfavorecedora para los sistemas educativos, ya que a tempranas edades, los niños y jóvenes comenzaban a salirse de la escuela (CabreraHernández, 2017; Kabay, 2016; Lyonga y Fosso, 2018) por varias razones; entre las más destacadas la pobreza, la falta de apoyo de los padres de familia y el rezago educativo (Colín, 2002).

Por lo anterior es que en México a través de una política pública, la Secretaría de Educación Pública (SEP) instancia encargada de brindar acceso a la educación a los individuos en conjunto con la Cámara de Diputados, quienes fungen de creadores de tales normativas, decidieron en el año 2012 promulgar la modalidad de acreditación automática para los niveles de Preescolar, Primaria y Secundaria pues ahí se registraban los mayores índices de abandono escolar. No obstante, no se previeron a futuro las posibles problemáticas que podrían desencadenarse en los estudiantes como aumento de las deficiencias en los logros estudiantiles, conformismo o desinterés familiar hacia los estudios de los hijos (Corzo y Reyes, 2017).

\section{La Educación Primaria en México}

Aunque la Educación Básica y elemental de México la constituyan el Preescolar (tres años), Primaria (seis años) y Secundaria (tres años), es en el nivel Primario en el que la mayor parte de los ciudadanos dejan de estudiar, logrando su culminación e incluso 
algunos abandonándola a mitad de camino (Instituto Nacional para la Educación de los Adultos, 2019).

La Educación Primaria se considera como primordial pues es en este nivel en el que los estudiantes aprehenden habilidades e información básica por cada una de las principales áreas del conocimiento; como lo son la expresión oral y escrita, las ciencias, matemáticas y artes. En México la Educación Primaria consta de seis años y a ella asisten niños desde los seis hasta los doce años.

Es en este nivel educativo en el que se encuentra la mayor producción tanto documental como empírica a contexto internacional, que se encarga de analizar los efectos académicos que se pueden registrar en los estudiantes al ser promovidos de manera automática. Aunque no se tienen registrados con profundidad cuáles son las respuestas actitudinales de los estudiantes al saber de su imposibilidad de reprobar o cuando ya se encuentran promovidos a un grado siguiente a causa de la normatividad.

\section{Opiniones docentes sobre la PA}

Los profesores externan en su mayoría estar en desacuerdo ante la PA pues enfatizan en que las habilidades académicas así como los conocimientos de los estudiantes merman su desarrollo y fortaleza como consecuencia del tránsito de año escolar sin la comprobación de sus aprendizajes (Feathers, 2020). Los docentes relatan también que aunado a ello, los estudiantes suelen manifestar conductas poco favorables hacia el aprendizaje; entre ellas destaca el mínimo esfuerzo (Pérez, et al., 2017) que incluso en la cultura mexicana se ve reflejada, debido a que este país por muchos años ha sido considerado como una nación que se conforma, es decir, que parte de sus ciudadanos se limitan a realizar el mínimo esfuerzo en sus actividades cotidianas, sin considerar si las ejecutan adecuadamente. Solo las culminan por cumplir y esto se está haciendo manifiesto en el desempeño escolar o actividades ejecutadas por los estudiantes del nivel primaria (Pérez, 2017).

Asimismo, la importancia de las opiniones docentes no ha obtenido la consideración por parte de las autoridades educativas de distintos países como las naciones africanas o asiáticas, dado que en palabras de Chohan y Qadir (2011) destacan que sus participaciones e ideas no son tomadas en cuenta para el desarrollo de políticas educativas que se ajusten a las problemáticas del país de manera profunda. Ni siquiera los profesores poseen la autoridad de decidir quienes promueven por méritos y quienes deben volver a recursar. Aunque en el contexto europeo de Rathman, et al., (2020) los profesores si 
tienen la libertad de emitir un fallo por los alumnos que deben promover o permanecer un año más en cierto grado de la educación primaria.

Aunque las opiniones docentes difundidas en torno a las actitudes del estudiantado, sean casi inexistentes, a continuación se recopilan algunas de ellas que aunque no son profundas, constatan la evidencia de particularidades conductuales por parte de los discentes ante la PA.

\section{Revisión de la literatura}

Se han encontrado algunas contribuciones en torno a las actitudes que refleja el alumnado ante una PA; en primera instancia, González-Pulido, et al., (2019) encontraron que los alumnos que cumplían de manera organizada con las actividades marcadas por el centro educativo, se sintieron desmotivados al ver que sus compañeros que no se esforzaban, obtenían las mismas recompensas que ellos en el contexto colombiano. Esta idea también la expresaron docentes en el estudio de Pérez, et al., (2017) en México y años previos en los trabajos de Owings y Kaplan (2001); Koppensteiner (2011); James (2017) y Pinzón (2018) en Estados Unidos, Brasil y Colombia. Dado que la PA no contribuye a la realización de un esfuerzo intelectual e incita a ejecutar actividades de baja calidad en el transcurso de un año lectivo. La creatividad de los alumnos se ve mermada e incluso los mismos profesores no toman en serio la evaluación, por tanto, lleva a un desajuste desalentador del alumno respecto de sus habilidades.

El estudio de Namen (2019) retrata con exactitud, transcurridos los años lo encontrado por sus pares en el 2001 esto porque detectó que los alumnos al conocer los beneficios de la PA, disminuyeron su nivel de esfuerzo para obtener una mejor calificación. Pese a esto, Anagnostopoulos en el 2006 tuvo un interesante hallazgo que difirió a los anteriores, ya que mostró que los alumnos repetidores fueron un incentivo para el interés en el aprendizaje de aquellos estudiantes de un ciclo, dado que representaron todo lo que no querían llegar a convertirse a futuro.

Ahmed y Mihiretie (2015) así como Rathman et al., (2020) encontraron en Etiopía y Alemania respectivamente, que los alumnos promovidos de manera automática manifestaron problemas de conducta, actitudes negativas hacia la escuela y menor atención en la realización de sus deberes académicos. Estos comportamientos incluso se hacen notorios en los estudiantes que se encuentran en riesgo de la repetición (Jimerson, et al., 2006). 
En la misma línea, la PA también genera una sensación de asombro, actitudes violentas por parte de los estudiantes hacia sus compañeros y otras conductas negativas que no se especifican. Pese a esto, suele ser un efecto temporal porque posteriormente se vislumbran sus mejoras académicas y actitudinales (Rathman, et al, 2020 y Haidary, 2013).

Refiriendo a situaciones opuestas a las enunciadas, Jimerson, et al., (2006) encontraron que la repetición no representa una situación que preocupe o resulte estresante al alumnado, dado que resultarían más estresantes otros eventos en su vida como perder la vista o algún padre.

García-Pérez, et al., (2011) hallaron que la repetición no baja la autoestima a los alumnos, por el contrario, los hace convertir su inmadurez hacia la preocupación por las actividades escolares, adquiriendo una mejor conciencia hacia sus aprendizajes.

Pese a estas aportaciones, de igual manera se pueden encontrar ideas que apoyen la PA. Aunado a ello Ahsan, et al., (2018) registraron mayor motivación del alumnado de la India ante la política de promoción automática pues consideraron que ya no existían presiones para ellos durante su proceso formativo, dejando de sentir estrés por una posible repetición, reflejando los propósitos nacionales por las que tal ley que promueve esta modalidad de acreditación, fue puesta en marcha. A mediano plazo esta motivación persistió en el alumnado y trajo consigo resultados favorables.

Schwerdt, et al., (2017) señalaron que las consecuencias negativas de una promoción de grado, pueden tardar en manifestarse unos años después y a pesar de esto, el rendimiento académico de los alumnos se mantendrá bajo sin poder recuperarse con el paso del tiempo.

Si se habla en comparación del sexo, los hombres suelen presentar un mayor índice de repetición escolar que las mujeres (Haidary. 2013 y Sunny, et al., 2017) esto desde los grados de primero a sexto año en la Educación Primaria en Malawi. De la misma forma Sunny y colaboradores identificaron que es más común la repetición en los grados de primero a tercer año de primaria y conforme se avanza en el trayecto escolar, tiende a decrecer. En la misma línea Leighton, et al., (2019) justifican sus resultados similares. Como se presenta, existen contribuciones que brindan indicios sobre las actitudes que los estudiantes pueden hacer manifiestas ante la PA, es por ello que a continuación se describe la manera en que se recopilaron los datos para este trabajo de investigación. 


\section{MATERIALES Y MÉTODOS}

El trabajo se desarrolló bajo el enfoque cualitativo y con el paradigma constructivista. Al tratarse de una comunidad educativa específica y para evitar la generalización se adoptó la técnica de estudio de caso singular descriptivo pues se limita a dar a conocer el fenómeno desde la mirada de un grupo de personas en particular que comparten centro de trabajo (Pérez Serrano, 1994). El estudio parte de un proyecto investigativo más amplio.

Los datos fueron obtenidos a través de entrevistas semi estructuradas durante el mes de septiembre del presente, para las cuales, los guiones empleados fueron previamente validados con personal experto en su elaboración. En tales guiones también se indagaron aspectos emocionales y académicos de los estudiantes que atraviesan la modalidad de acreditación que se estudia. Se contó con la participación de ocho docentes y de la directora de una escuela primaria pública ubicada al norte de Mérida, Yucatán, México, los cuales a través de sus experiencias dieron respuestas a los cuestionamientos. A continuación se muestra su información básica.

\section{Tabla 1}

Información de los participantes

\begin{tabular}{cccc}
\hline Actor educativo & Género & Grado & Años de servicio \\
\hline Directora & Mujer & N/A & Más de 15 \\
G & Hombre & $1^{\circ}$ & Menos de 3 \\
H & Mujer & $1^{\circ}$ & Entre 3 y 5 \\
A & Mujer & $2^{\circ}$ & Más de 15 \\
B & Mujer & $2^{\circ}$ & Entre 3 y 5 \\
C & Mujer & $3^{\circ}$ & Más de 15 \\
D & Hombre & $4^{\circ}$ & Entre 3 y 5 \\
E & Mujer & $5^{\circ}$ & Entre 3 y 5 \\
F & Hombre & $6^{\circ}$ & Entre 3 y 5 \\
\hline
\end{tabular}

La duración de las entrevistas fue de entre los 11 hasta los 27 minutos y una de las cuestiones precisamente se enfocó a que los profesores brindaran información en cuanto a las actitudes de los alumnos ante la PA. El propósito de la entrevista fue presentado y los participantes tuvieron acceso a un documento sobre sus derechos y obligaciones 
durante el trabajo de investigación. Posterior a ello, se les proporcionó una carta de consentimiento informado en la que autorizaban la grabación de su voz para posteriormente transcribir sus respuestas y de esta manera realizar los análisis.

Una vez realizadas las transcripciones, se procedió a separar las respuestas de los participantes. De manera particular se identificaron las actitudes y fueron enlistadas en una tabla a doble columna en la que se hizo señalamiento del docente que brindó determinado comentario.

\section{Limitaciones}

$\mathrm{Al}$ tratarse de una escuela en específico y considerando que no todos los profesores de la planta docente accedieron a participar en la investigación, no es posible generalizar los resultados que aquí se exponen. A su vez no se puede decir que tales actitudes persisten para la diversidad de contextos escolares existentes. Esto es debido a que las respuestas brindadas por los actores educativos son consecuencia de experiencias diversas que han experimentado dentro de la escuela en la que laboran así como de otras en la que han trabajado en la misma ciudad o en localidades aledañas del Estado. Asimismo, el dar a conocer exclusivamente las actitudes del alumnado ante la PA, no permite comprender a profundidad los contextos de los cuales los estudiantes proceden a causa de tales conductas y de entender a quienes brinda mayores beneficios la normativa de acreditación automática.

Además el trabajo de investigación no consideró las opiniones procedentes directamente del alumnado y tampoco contempló observaciones en el ambiente de aprendizaje.

\section{RESULTADOS Y DISCUSIÓN}

Los ocho profesores y la autoridad educativa del plantel participante manifestaron la diversidad de actitudes en los estudiantes. Para ello, se mostrarán parte de los resultados organizados por los autores en la Tabla 2. 


\section{Tabla 2}

Efectos actitudinales de la política de PA en el alumnado

\begin{tabular}{|c|c|c|}
\hline $\begin{array}{c}\text { Actitudes derivadas del } \\
\text { desinterés }\end{array}$ & $\begin{array}{c}\text { Actitudes que son } \\
\text { generadas por el miedo }\end{array}$ & $\begin{array}{c}\text { Actitudes que } \\
\text { generan violencia }\end{array}$ \\
\hline $\begin{array}{l}\text { a) No hay esfuerzos por parte } \\
\text { del alumnado hacia una } \\
\text { actividad }\end{array}$ & a) Apatía & a) Bullying \\
\hline b) Irresponsabilidad & b) Aislamiento & b) Conductas agresivas \\
\hline $\begin{array}{l}\text { c) Repetición de los mismos } \\
\text { patrones conductuales del } \\
\text { ciclo escolar previo }\end{array}$ & $\begin{array}{l}\text { c) Rechazo a las actividades } \\
\text { escolares }\end{array}$ & del alumno promovido \\
\hline d) Conformismo & d) Actitudes de derrota & \\
\hline e) Cinismo & e) Inseguridad & \\
\hline $\begin{array}{l}\text { f) Manipulación por parte de } \\
\text { los alumnos hacia sus } \\
\text { padres y maestros }\end{array}$ & f) Dificultades para socializar & \\
\hline $\begin{array}{l}\text { g) Rechazo a las actividades } \\
\text { escolares }\end{array}$ & g) Bloqueo cognitivo & \\
\hline h) Aburrimiento & h) Desmotivación & \\
\hline i) Seguridad & i) Dificultades para socializar & \\
\hline j) Rechazo & $\begin{array}{l}\text { j)Incomodidad } \\
\text { mismos }\end{array}$ & \\
\hline
\end{tabular}

Nota: Elaboración propia.

Tales actitudes se clasificaron de acuerdo a sus características en común y a la información recabada de la literatura existente.

Las actitudes que desencadenan el miedo y el desinterés son las que predominan, considerando que se pueden llegar a manifestar tanto en los alumnos que han sido promovidos mediante esta modalidad de acreditación automática, como en aquellos que se encuentran en riesgo de recursar un año escolar. En contraparte las actitudes agresivas son las que menos prevalecen, sin embargo, no se descarta su gravedad.

Generalmente las actitudes vinculadas al miedo suelen manifestarse con más frecuencia en alumnos de los primeros tres grados de la primaria y las actitudes derivadas del desinterés y la violencia provienen de estudiantes que cursan los grados del $4^{\circ}$ al $6^{\circ}$. 
A continuación se recopilan algunas aportaciones de los actores educativos que contribuyeron a la realización de la Tabla 2:

“...cinismo. Porque ya se sabe que el alumno va a pasar sin esfuerzo.

Con el solo hecho de estar inscrito en la escuela, ya tiene su pase automático (...) ya no hacen ningún esfuerzo, ya no se aplican, ya no se esfuerzan..." Directora

"Hay algunos alumnos que llegan, listos y tal cual te dicen, total voy a pasar, no toman el interés por venir a la escuela. Vienen porque los mandan (...) saben que tienen que pasar a segundo..." Profesora " $\boldsymbol{H}$ "

Tal aportación la comparte la docente "E" en la que los mismos padres son quienes no le prestan la atención debida a los estudios de sus hijos:

(...) llevo a mi hijo a la escuela porque lo tengo que llevar, porque es la norma social, de que yo tengo que llevar a mi hijo a la escuela, por lo menos a Primaria, pero no los llevan para que aprendan, los llevan porque tengo que llevarlo y así me lo cuidan de 7 a 12, pero no les interesa en realidad..."

Aunque también se identifica lo siguiente y que precisamente se asocia al miedo de ser promovido o de haber sido parte de esta acreditación:

(...) al pedirle alguna participación, y ver que otros niños pueden y él o ella no, eso va creando inseguridades en el alumno. Aunque le digamos "si puedes, tu puedes y si no, te ayuda el compañero, no pasa nada" pero el alumno se da cuenta de que no puede. Profesora "B"

Se relataron también actitudes de violencia en los alumnos que suelen recursar. Sin embargo tales actitudes violentas no proceden de ellos, sino de sus compañeros de clase. Entre las aportaciones de los profesores del estudio se hallaron:

“...el niño (...) podría ser víctima de burlas tal vez, bullying, muchos aspectos contraproducentes" Profesor “G”

El profesor "F" también señala:

“(...) se sienten mal consigo mismos porque pueden sufrir burlas”

Por el aspecto de los alumnos que suelen ser violentos con los profesores al saber de su pase automático, se obtuvo lo siguiente también por parte del profesor "F" quien ha 
trabajado los grados del $5^{\circ}$ al $6^{\circ}$ :

“...presentan conductas agresivas, desinterés, apatía (...) los niños son inteligentes ya saben si hice esto y no pasó nada..."

Como se evidencia, se han recabado respuestas variadas entre el colectivo docente y la mayor parte se dirigen hacia manifestaciones desfavorables por parte de los alumnos hacia la escuela, algunas de ellas transmitidas por los mismos tutores. También dichas conductas apuntan a ocasionar miedos e inseguridades que poco contribuyen a la motivación o mejora de los aprendizajes de los estudiantes.

Como lo presentaron Ahmed y Mihiretie (2015) así como Rathman et al., (2020) los estudiantes que promueven automáticamente, suelen presentar actitudes negativas hacia las actividades escolares además de presentar mayor desinterés hacia los estudios, lo cual se vio reflejado en los comentarios emitidos por los profesores. No obstante, también se encontraron conductas violentas en los alumnos promovidos, aunque en este estudio no fueron de mayor frecuencia como en los trabajos enmarcados en la literatura de la temática. Sin embargo se descubrió que las actitudes violentas proceden en mayor medida de los compañeros de curso hacia los estudiantes promovidos al identificar sus desaciertos en cuanto a habilidades y conocimientos, esto se pudo identificar a través de las aportaciones de los actores educativos que participaron.

Asimismo, los resultados compaginaron a las aportaciones de Namen (2019); Koppensteiner (2011) y Pinzón (2018) pues los docentes destacaron que al conocer los estudiantes la imposibilidad de la reprobación, disminuyen su esfuerzo intelectual, no se permiten ser creativos y aminoran su disposición así como su apertura para el aprendizaje. Por lo que se le pueden relacionar la apatía y el conformismo.

Los resultados recabados por Jimerson, et al., (2006) y García-Pérez, et al., (2011) difieren de los hallazgos en este estudio. Mientras estos autores relataron que un recursamiento es algo que poco estrese a los estudiantes; en palabras de algunos de los docentes participantes, los alumnos desarrollan inseguridades y autoestima baja al enterarse que pueden ser víctimas de un recursamiento. Incluso ya aprobados de manera automática, cuando identifican que no llevan el mismo ritmo de trabajo que sus compañeros de grupo, se ven desesperados, desmotivados y desarrollan un bloqueo cognitivo, lo que conlleva a un aislamiento social, haciéndolos sentir poco capaces de relacionarse con otros y de realizar sus tareas académicas. Los resultados también son 
contrarios a lo enunciado por Ahsan et al., (2018) a causa de que mientras los niños participantes de su proyecto presentaron mayor motivación al ser acreditados de manera automática, los docentes participantes de la presente investigación expresaron que la motivación es lo que menos persiste entre el alumnado que sabe que recursará y en aquellos que promovieron de manera instantánea, pues ya están con una actitud de derrota incluso antes de volver a intentar algo.

\section{CONCLUSIONES}

- La PA es una modalidad de acreditación cuyo propósito es evitar el abandono escolar del alumnado debido a sus condiciones económicas y sociales, las cuales no puede controlar. Pese a su buena intención, no contempla indicios que a largo o mediano plazo, pueden obstaculizar el trayecto académico del alumnado, pero que también pueden influir en sus actitudes de aceptación o rechazo a las actividades escolares y a su desempeño intelectual.

- Lamentablemente predominan diversidad de actitudes negativas al saber que no existe la posibilidad de reprobación aunque los mismos estudiantes tengan la conciencia y conocimiento de no dominar ciertos contenidos o habilidades de un grado determinado. Lo que los hace demostrar desinterés, irresponsabilidad y apatía hacia sus estudios, siendo predominante entre las actitudes de los alumnos que cursan del $4^{\circ}$ al $6^{\circ}$ grado de la educación primaria.

- Por su parte, los niños que estudian del $1^{\circ}$ al $3^{\circ}$ suelen sentir mayor inseguridad, miedo y desmotivación al momento de ejecutar sus tareas escolares.

- Estas actitudes pueden manifestarse antes de recibir un veredicto de promoción automática o una vez ya promovidos al siguiente grado escolar.

- Los profesores señalan que parte de las actitudes que los alumnos desarrollan, proceden de lo que sus padres hablan en el hogar. Por tanto son también los tutores, quienes propician tales conductas o pensamientos en los niños, los cuales los van incitando a una cultura del mínimo esfuerzo, restringiendo su seriedad y compromiso hacia los estudios, aprovechándose de la normatividad que no permite la reprobación.

\section{CONSIDERACIONES FINALES}

Es oportuno mencionar que los resultados no exploran de manera directa las opiniones del estudiantado, por lo que se puede realizar un trabajo cualitativo que ahonde en la observación e identificación áulica de las actitudes de alumnos que han promovido de 
manera automática. También es preciso destacar la elección de un caso en particular de un alumno o alumnos que permitan conocer a mediano y largo plazo su desenvolvimiento actitudinal ante las actividades que se realizan en la escuela así como la disposición de sus tutores para con los deberes académicos de sus hijos.

Valdría la pena de igual manera extender la recopilación de datos por parte de otros agentes educativos en distintos puntos geográficos de la ciudad, pues en cada zona se viven situaciones sociales y económicas distintas. A través de ello, podrían segmentarse las opiniones de profesores y realizar otro estudio que únicamente incorpore directores.

En el contexto nacional y local debido a que la normatividad se encuentra en vigencia, es necesaria la proliferación de estudios empíricos que permitan comprender y hacer visibles los efectos que una normativa está generando en los estudiantes de educación Primaria. Asimismo, es importante realizar un trabajo siguiendo las recomendaciones ya enlistadas en los niveles de preescolar y secundaria, pues junto a la primaria, constituyen la educación formativa básica para los niños mexicanos.

Por último, la investigación de las actitudes en el alumnado en los contextos cuya normatividad de PA se encuentra en vigencia, permitirá una comparación entre regiones distintas para encontrar coincidencias o discrepancias con los resultados ya expuestos.

\section{Apoyos}

Este trabajo ha sido posible gracias a la concesión de efectivos a través de la beca número 1089895 del Consejo Nacional de Ciencia y Tecnología (CONACYT).

\section{REFERENCIAS BIBLIOGRAFICAS}

Ahmed, A. y Mihiretie, D. (2015). Primary school teachers and parents' views on automatic promotion practices and its implications for education quality. International Journal of Educational Development, 43(2015), 90-99. http://dx.doi.org/10.1016/j.ijedudev.2015.05.003

Ahsan, M. Banerjee, R. y Hari, S. (2018). Social Promotion and Learning Outcomes: Evidence from India. USC-INET Research Paper, sin vol(18-14), 1-44. http://dx.doi.org/10.2139/ssrn.3256387

Anagnostopoulos, D. (2006). "Real Students" and "True Demotes": Ending Social Promotion and the Moral Ordering of Urban High Schools. American Educational Research Journal, $43(1)$, $5-42$. https://journals.sagepub.com/doi/10.3102/00028312043001005 
Cabrera-Hernández, F. (2017). Leave them kids alone! The positive effect of abolishing grade retention on pupils' dropout rates: Evidence from a policy change. Centro de Investigación y Docencia Económicas. https://cinst.hse.ru/data/2019/12/08/1521175504/Cabrera\%20(2017).pdf

Chohan, B. y Qadir, S. (2011). Automatic Promotion Policy at Primary Level and MDG2. Journal of Research and Reflections in Education, 5(1), 1-20 https://www.researchgate.net/publication/216035878_Automatic_Promotion_Po licy_at_Primary_Level_and_MDG-2

Colín, M. (2002). Factores de reprobación y bajo rendimiento escolar, en alumnos de la escuela secundaria oficial no. 6" José María González Arratia" turno vespertino". [Tesis de Maestría. Instituto Tecnológico y de Estudios Superiores de Monterrey]. Repositorio del Tecnológico de Monterrey https://repositorio.tec.mx/bitstream/handle/11285/632012/EGE0000001831.pdf? sequence $=1$

Corzo, C. y Reyes, C. (2017). Principales causas de reprobación de alumnos de los grupos de quinto semestre grupo seis y ocho de la escuela preparatoria número tres. (Capítulo antecedentes). https://repository.uaeh.edu.mx/revistas/index.php/prepa3/article/download/2079/ 5386? inline $=1$

Delgado, P. (2019, 21 de octubre). La importancia de la participación de los padres en la enseñanza. Instituto para el Futuro de la Educación. Observatorio. https://observatorio.tec.mx/edu-news/la-importancia-de-la-participacion-de-lospadres-en-la-educacion

Feathers, C. (2020). Teacher and Administrator Beliefs about Grade Retention in Northeast Tennessee School Districts. [Tesis de Doctorado. East Tennessee State University]. Repositorio de la Universidad del Este del Estado de Tennessee. https://dc.etsu.edu/etd/3704/

García-Pérez, I. Hidalgo-Hidalgo, M. y Robles, J. (2011). Does grade retention affect achievement? Some evidence from PISA. Working Papers, 37(2011). Universidad Pablo de Olavide, Department of Economics. http://www.upo.es/serv/bib/wps/econ1109.pdf 
González-Pulido, J. González-Pulido, A. y Cifuentes, J. (2019). La reforma educativa y la calidad de la educación en Colombia. Revista Humanismo y Sociedad, 7(2), 4153. https://doi.org/10.22209/rhs

Haidary, A. (2013). Controvercy over grade repetition. Afghan Teachers' View on Grade Repetition. [Tesis de Maestría. Universidad de Karlstad]. International Household Survey Network. http://www.divaportal.org/smash/get/diva2:695166/FULLTEXT01.pdf

James, T. (2017). The effect of automatic promotion on performance of learners in primary schools in Mubende District. [Reporte de investigación. Kampala International University]. Kampala International University. Research and Archives. https://ir.kiu.ac.ug/handle/20.500.12306/2551

Jimerson, S. Pletcher, S. y Graydon, K. (2006). Beyond grade retention and social promotion: promoting the social and academic competence of students. Wiley Periodicals, $43(1)$, $85-97$. https://www.researchgate.net/publication/227786160_Beyond_grade_retention_ and_social_promotion_Promoting_the_social_and_academic_competence_of_st udents

Kabay, S. (2016). Grade Repetition and Primary School Dropout in Uganda. Harvard Educational Review, 86(4), 580-606. https://eric.ed.gov/?id=EJ1123785

Koppensteiner, M. (2011). Automatic grade promotion and student performance: Evidence from Brazil. University of Leicester. https://hdl.handle.net/2381/33125

Leighton, M. Souza, P. y Straub, S. (2019). Social Promotion in Primary School: Effects on Grade Progression. Brazilian Review of Econometrics, 39(1), 1-33. http://bibliotecadigital.fgv.br/ojs/index.php/bre/article/view/78513/76362

Lyonga, N, y Fosso, N. (2018). Effects of Collective Promotion on the Attainment of Goals of Basic Education in English-Speaking Primary Schools in Cameroon. Journal of Science and Education, 44(2), 259-272. http://dx.doi.org/10.21796/jse.2020.44.2.259

Namen, O. (2019). The Impact of Encouraging Social Promotion. http://dx.doi.org/10.2139/ssrn.3402191

Ortega, N., García, R., Guerra, Y., Álvarez, A., Hérnandez, N., (2010). Actitudes de los niños de entre 9 y 12 años de edad, ante problemas familiares. Revista Científica 
Electrónica de Psicología, sin vol(10), $\quad$ 55-69. https://www.uaeh.edu.mx/investigacion/productos/2181/

Owings, W. y Kaplan, L. (2001). Stardards, retention and social promotion. Nacional Association of Secondary School Principals, 85(629), 57-66. https://doi.org/10.1177/019263650108562906

Paz, Octavio (1990). El Laberinto de la Soledad. Fondo de Cultura de Económica.

Pérez, H. (2017). Percepción docente sobre los criterios de acreditación y promoción de los alumnos de Educación Primaria. [Tesis de maestría. Universidad Autónoma de Yucatán]. Repositorio de trabajos de titulación de la Facultad de Educación. http://tesis.educacion.uady.mx/

Pérez, H. Aguilar, A. y Heredia, N. (2017, 22 de noviembre). Propuesta de mejora docente de los lineamientos de acreditación y promoción de alumnos de educación primaria. [ponencia] XIV Congreso Nacional de Investigación Educativa, San Luis Potosí, México. http://www.comie.org.mx/congreso/memoriaelectronica/v14/doc/1249.pdf

Pérez Serrano, G. (1994) Investigación cualitativa. Retos, interrogantes y métodos. La Muralla.

Pinzón, D. (2018). Reprobación y desempeño académico: Evidencia de la implementación de la promoción automática en Colombia. Documentos CEDE, sin vol(18), 1-41. http://dx.doi.org/10.2139/ssrn.3157825

Rathman, K. Lother, K. y Vockert, T. (2020). Critical Events throughout the Educational Career: The Effect of Grade Retention and Repetition on School-Aged Children'sWell-Being. International Journal of Environmental Research and Public Health, 17(11), 1-30. https://doi.org/10.3390/ijerph17114012

Razeto, A. (2016). El involucramiento de las familias en la educación de los niños. cuatro reflexiones para fortalecer la relación entre familias y escuelas. Revista Páginas de Educación, 9(2), 1-26. http://www.scielo.edu.uy/scielo.php?pid=S1688$74682016000200007 \&$ script=sci_abstract

Schwerdt, W. West, M. y Winters, A. (2017). The Effects of Test-based Retention on Student Outcomes over Time: Regression Discontinuity Evidence from Florida. Journal of Public Economics, 152(2017), 154-169. https://doi.org/10.1016/j.jpubeco.2017.06.004 
Sunny, B. Elze, M. Chihana, M. Gondwe, L. Crampin, A. Munkhondya, M. Kondowe, S. y Glynn, J. (2017). Failing to progress or progressing to fail? Age-for-grade heterogeneity and grade repetition in primary schools in Karonga district, northern Malawi. International Journal of Educational Development, 52(2017), 68-80. http://dx.doi.org/10.1016/j.ijedudev.2016.10.004

Vallejo-García, F. (2018). Criterios de promoción estudiantil como factores conexos al éxito o fracaso escolar. Revista Latinoamericana de Estudios Educativos, 14(1), 1-19. https://www.redalyc.org/articulo.oa?id=134156702004 